\title{
La política económica europea vista desde el Estado español
}

\author{
Antoni Seguí-Alcaraz \\ Universitat de València
}

\section{Resumen}

Este análisis revisa la posición española sobre la reforma de la gobernanza económica de la zona euro. Analizamos el marco normativo y operativo que impuso la Unión Monetaria Europea (UME) a sus miembros y el principal motivo por el que no fue eficaz en prevenir las recientes crisis nacionales.

Por otra parte, se lleva a la práctica una valoración del desarrollo del pacto de estabilidad y crecimiento, con el objeto de valorar las políticas económicas aplicables desde España y el empleo. Observamos un reñido debate en los países miembros, ya que el proceso hace necesario aunar posiciones políticas muy diferentes.

Palabras clave: pacto de estabilidad, Consejo, semestre europeo, sector financiero, empleo.

Clasificación JEL: D52, E52, P16.

\begin{abstract}
This analysis reviews the Spanish position on the economic governance reform inside the euro area. We analyze the regulatory and operational framework imposed by the European Monetary Union (EMU) to its members and the main reason why it was not effective in preventing the recent national crisis.

On the other hand it takes an assessment on the development of the stability and growth development pact, in order to assess the relevant, applicable economic policies from Spain and its employment. We observe a heated debate between member countries, since this process makes it necessary to combine very different political positions.
\end{abstract}

Keywords: stability pact, Council, European semester, financial sector employment.

JEL classification: D52, E52, P16.

\section{Introducción}

La reciente crisis financiera ha exigido fuertes medidas de apoyo financiero, pero sobre todo ha destapado la falta de un adecuado Pacto de Estabilidad y Crecimiento, lo mismo para verificar procesos presupuestarios desordenados que para hacer frente a perturbaciones surgidas en el sector privado. La envergadura de las dificultades explica que las reacciones dentro de la Unión fueran poco coherentes en un principio, pero a lo largo del último lustro el país de mayor peso, Alemania, definió su postura política y actuó en apoyo tanto de la Unión como de sus miembros.

Se habla de un sistema de gobierno para la economía de la eurozona y de un pacto de competividad. De momento, es difícil que la Unión Monetaria Europea (UME) 
pueda ir más allá de soluciones pragmáticas, sin transformaciones de fondo en la estructura jurídico-política de la Unión ni cesiones significativas de soberanía. Avanzar hacia modalidades de federalismo fiscal puede encontrar resistencia en el Tribunal Constitucional alemán y tal vez en algunas capitales que hasta hoy no se han pronunciado. Puede resultar interesante que España pueda apoyar un nuevo marco más coherente para la Unión Monetaria Europea, introduciendo mecanismos de contraste de sus políticas con las obligaciones asumidas y acelerando su convergencia real con los países más avanzados.

El objeto de este trabajo es realizar una valoración de la política económica europea, desde España. El análisis empieza haciendo una reflexión en torno al marco normativo y operativo que originalmente impuso la unión monetaria a sus miembros y el motivo por el que no fue eficaz en prevenir las recientes crisis de los países de la unión.

\section{Orígenes de la Unión Monetaria Europea}

La integración europea no fue obra de visionarios teóricos, sino de políticos realistas. Ha tenido una trayectoria de marcado pragmatismo, dando margen para que consecutivos logros, a veces con cierta lentitud, se asimilaran por los miembros y fueran adquiriendo suficiente madurez. Además, han vivido etapas de entusiasmo arrollador, en que políticos impacientes han preferido olvidar la importancia de una consolidación gradual y acelerado objetivos cuyo cumplimiento necesitaba todavía complejas adaptaciones institucionales. La unión monetaria ha sido uno de estos casos (ciertamente no el único). La unión monetaria empezó encuadrada en un marco normativo y operativo, el Pacto de Estabilidad y Crecimiento, indiscutiblemente defectuoso.

El proyecto de unión monetaria quedó abierto a países miembros de la Unión Europa, cuyas estructuras económicas eran sumamente dispares. Según la literatura económica de los años sesenta, en particular el trabajo de Mundell (1960), un área monetaria difícilmente será óptima en esas condiciones porque los límites de tolerancia a las tensiones impuestas por la seriedad de una moneda única serán muy distintos en economías heterogéneas. Esta reflexión marcaron durante años las prevenciones alemanas al proyecto. Era lógico que la opinión pública alemana nunca aceptaría desprenderse de una moneda estable y sólida, siempre que el proyecto pudiera traer riesgos del desorden monetario fiscal y que durante muchos años había caracterizado las políticas económicas de Italia o Francia, además de países como Portugal o España, que en un primer momento ni siquiera parecían candidatos con posibilidades. Por ese mismo motivo, el gobierno de Kohl subordinó su conformidad a la creación de un Banco Central Europeo a imagen del Bundesbank, así como al cumplimiento de una serie de criterios de política económica primero en la fase de entrada y posteriormente durante el desarrollo del proyecto. Por otra parte, se pensó que la estabilidad monetaria y el aumento de los flujos comerciales intraunión mo- 
netaria traerían importantes mejoras de productividad a las economías más frágiles e impulsarían, por tanto, su proceso de convergencia real (no hace falta insistir en que este optimismo resultó injustificado).

Los criterios «de convergencia» son conocidos por todos y basta ahora con recordar los máximos de un 3 por 100 de déficit y un 60 por 100 en deuda pública externa (Comisión Europea, 2008). Dos observaciones me parecen relevantes.

Los análisis económicos han hecho hincapié en las disparidades o semejanzas estructurales de las economías interesadas en formar un área monetaria. Hay que decir que la «convergencia» de que hablan Maastricht y el Pacto no es estructural, sino que propone solamente homogeneizar datos coyunturales críticos de las economías participantes (por ejemplo, la tasa de desempleo no sea uno de los criterios). Los países aspirantes más débiles hicieron un gran esfuerzo por cumplir con las condiciones de acceso, y aceptaron someter sus políticas nacionales de crecimiento a un marco monetario supranacional y a criterios limitativos en materia fiscal. Pertenecer a la unión monetaria permitía compartir una experimentada política de estabilidad económica a países que durante años jugaron despreocupadamente con inflación y devaluaciones. En términos políticos y económicos, este es un beneficio extraordinario (Comisión Europea, 2009).

Por otra parte, llega solamente hasta un punto, porque, en cambio, participar en el proyecto de moneda única no otorga facilidades especiales para salvar la distancia entre estructuras económicas. Las economías menos fuertes tienen que identificar su propia senda de crecimiento económico. La presión francesa para modificar el etiquetado del Pacto -añadiendo «Crecimiento» a «Estabilidad»- fue simple cosmética y dejó intacta la filosofía esencial del Pacto (Deroose et al., 2008).

Además, el Pacto, plegándose al temor alemán -más que justificado- a los desmanes fiscales de algunos candidatos, no tuvo en cuenta la posibilidad de que déficit presupuestario y deuda creciente fueran consecuencia de crisis económicas producidas por desmanes inversores del sector privado (o por impredecibles shocks externos como una crisis financiera mundial). El debate académico, en su día, se centró en aspectos mecánicos del Pacto, comprobando, por ejemplo, que un nivel de deuda del 60 por 100 es sostenible con déficit recurrentes del 3 por 100 siempre que la economía esté creciendo al 5 por 100 nominal.

Hemos de observar que el saldo de balanza por cuenta corriente de una economía es idéntico a la suma de saldos de ahorro/inversión privada y de gasto/recaudación del sector público. Por tanto, un déficit público -digamos, del 4 por 100- puede quedar sobrecompensado por un superávit del sector privado -digamos, un 9 por 100-, generando un saldo acreedor de la economía frente al exterior. Con ese dato fiscal, el Pacto activaría el procedimiento de déficit excesivo, pero cualquier analista interpretaría el déficit público en el contexto mucho más amplio de una economía ahorradora neta y con saldo positivo de cuenta corriente. Y al revés, aunque las cuentas públicas estén ordenadas, el sector privado puede estar desahorrando e incurriendo en un fuerte endeudamiento frente al exterior. Como consecuencia el saldo de la balanza de cuenta corriente reflejará esta disparidad de comportamientos, pero el Pacto 
no activaría sus alertas a pesar de la seria amenaza macro que se cierne sobre el país. En el caso de la burbuja inmobiliaria española: no se había violado el criterio del 3 por 100 desde la creación de la eurozona en 1999. Por otra parte, los sensores del Pacto estaban dispuestos para captar excesos del sector público, de tal manera que no reaccionaron delante de la acumulación de déficit y de financiación externa de un sector privado en plena euforia inversora. Decir que el Pacto ha mostrado críticas debilidades de funcionamiento. Así, careció de la sensibilidad necesaria para hacer frente a la manipulación de cifras de un país como Grecia, donde el historial en la unión monetaria europea estaba marcado por incumplimientos casi sistemáticos del criterio del 3 por 100 .

\section{Desarrollo del pacto de estabilidad y crecimiento}

Los cambios que incorporó el pacto a partir de su reformulación en 2005 han sido objeto de amplio análisis tanto por la propia Unión Europea como por parte del mundo académico. Aquí nos limitaremos a una breve exposición de los mismos, centrándonos especialmente en aquellos aspectos que han permitido la ejecución de políticas fiscales expansivas en la actual situación y que, bajo el formato original, difícilmente hubieran encontrado cabida (Carpio, 2004); de esta manera las principales modificaciones son:

Infringir el pacto no constituye novedad. Se incumplió abiertamente cuando Francia y Alemania cerraron sus cuentas públicas con déficit superior al 3 por 100 entre 2002 y 2005 . Fue un episodio muy poco edificante, porque los dos países más importantes de la unión monetaria europea consiguieron imponer enmiendas al Pacto en lugar de someterse al procedimiento sancionador previsto. La propia Comisión Europea actuó muy responsablemente, interponiendo un recurso -rechazado finalmente- contra la decisión del Consejo (Knappe y Weber, 2007).

Es forzoso admitir, de todos modos, que los déficit francés y alemán no transmitieron ninguna vibración significativa a la economía de la Unión Monetaria Europea. Ahora, en cambio, el estallido de las burbujas inmobiliarias irlandesa y española, el déficit público portugués y los déficit persistentes y manipulados de Grecia, han tenido efectos desbordamiento y han sacudido violentamente la cohesión dentro de la Unión Monetaria Europea. Cada uno de los países ha visto caer su calificación en el mercado financiero y soportado fortísimas oscilaciones de su prima de riesgo. Gobiernos y bancos han tenido dificultades de financiación -en diverso grado- y el riesgo de insolvencia soberana en países de la eurozona ha flotado en los ambientes financieros (Carpio, 2004). 
Parte correctiva. En general, se ha introducido una mayor flexibilidad en el procedimiento de déficit excesivo.

- Se define el término «recesión económica severa», que incluye tanto un crecimiento negativo del PIB como un crecimiento continuado por debajo del PIB potencial.

- Se introduce una relación de «otros factores pertinentes» que justifican la no apertura del procedimiento de déficit excesivo o son tenidos en cuenta por el Consejo en sus recomendaciones en los casos en que sí se lance el procedimiento (Mateos de la Nava, 2009). Estos factores no quedaban enumerados ni en el Tratado de Maastricht ni en el Pacto de Estabilidad y Crecimiento original.

- Se amplían los plazos para las distintas etapas del procedimiento de déficit excesivo, proporcionando un mayor margen de actuación a los Estados que incurran en déficit excesivo.

- Se tienen en cuenta los «acontecimientos especiales adversos» en el procedimiento de déficit excesivo, tanto en su lanzamiento como en su ejecución.

- Se incrementa la atención sobre la deuda y la sostenibilidad a medio plazo de las cuentas públicas, recuperando la idea del Tratado de una ratio deuda/PIB que presente una senda de disminución y aproximación al valor de referencia a un ritmo satisfactorio, aunque estos términos no son cuantificados .

\section{Parte preventiva}

- Cada Estado define su propio objetivo presupuestario a medio plazo (Medium Term Objective -MTO-), que es analizado y valorado por el Consejo. Este objetivo puede estar alejado de una posición de «equilibrio o con superávit», que era lo que fijaba la formulación inicial. No obstante, se establece un intervalo entre el -1 por 100 del PIB y «equilibrio o con superávit», para el saldo estructural. Es decir, las reglas fiscales impuestas por la Unión son complementadas ahora por reglas fiscales de los propios Estados. Al tomar como referencia el déficit estructural, se están teniendo en cuenta las condiciones cíclicas (Hüfner, 2004).

- Para el proceso de ajuste hacia el objetivo a medio plazo fijado por cada país, los Estados deberán perseguir una mejora anual de 0,5\% del PIB para el saldo estructural. El esfuerzo deberá ser mayor en épocas de bonanza.

- Los países pueden desviarse de su objetivo a medio plazo o de la senda de aproximación al mismo, si se debe a que acometen reformas estructurales que supongan un ahorro a largo plazo en los presupuestos, especialmente si se refieren al sistema de pensiones (Krugman, 2009). 
Las valoraciones de la reforma del pacto de estabilidad y crecimiento son de distinto signo, aunque se pueden resumir en las dos posiciones extremas siguientes (Hüfner, 2004). Para los defensores, con los cambios se ha logrado una mayor racionalidad del Pacto mediante una mejor adaptación de las reglas a las diferentes circunstancias económicas y necesidades de cada país, que ayudará al mejor cumplimiento de las obligaciones. Los detractores, por su parte, afirman que la reforma ha descafeinado el pacto de estabilidad y crecimiento con sus numerosas vías de escape en el procedimiento de déficit excesivo, con reglas más complicadas y menos transparentes en general, todo lo cual, en definitiva, ha representado una relajación de la disciplina fiscal (BCE, 2009).

En cualquier caso, su bondad dependerá de cómo se aplique. Lo cierto es que, atendiendo a las circunstancias actuales, el Pacto reformado está permitiendo a los países un mayor margen de maniobra para gestionar la crisis económica. Más concretamente, el deterioro de las cuentas públicas -déficit y deuda-, que es el resultado combinado de los estabilizadores automáticos y de los planes de estímulo fiscal, queda amparado por la presencia de «acontecimientos especiales adversos» que, aunque no impiden el inicio del Procedimiento de Déficit Excesivo -como se verá más adelante- sí posibilitan una gestión y secuencia temporal del mismo menos estricta para los Estados. Es decir, hay mayor tolerancia frente a déficit superiores al 3 por 100, como es el caso actualmente de numerosos países de la Unión (Carpio, 2004).

\section{La importancia de la regulación del sector financiero europeo}

Estamos percibiendo actualmente los inmensos costes que tiene tratar de limitar los daños producidos por la longitud y la severidad de la crisis. Se han llevado a cabo salvamentos de entidades financieras (bailouts), se ha relajado la política monetaria (quantitative easing), se han introducido estímulos y se han garantizado emisiones de deuda. Necesitamos, por tanto, diseñar mecanismos que reduzcan la probabilidad de que se vuelva a producir una nueva crisis financiera en el futuro. Una buena parte del problema ha estribado en la excesiva indulgencia con que hemos contemplado la posibilidad de una crisis financiera, dado el tiempo transcurrido desde la de Depresión de 1929 y la creencia en que el éxito en la resolución de recientes crisis menores probaba nuestra ilimitada capacidad para negociar con ellas (BCE, 2009).

Ha habido tres claros fallos de prevención: una excesiva desregulación, un descuido de algunos indicios de peligro, así como una excesiva indiferencia acerca del notable descenso en la tasa de ahorro y en el nivel de riesgo asumido por dicho ahorro. Para evitarlos, es importante que la regulación y supervisión no estén excesivamente fragmentadas; en Europa es fácil pensar en la conveniencia de una agencia europea de supervisión bancaria. Es asimismo importante que la información fluya entre las agencias responsables. Por el contrario, ahora hemos debido aprender que precisamos de planes contingentes por parte de los bancos centrales y los gobiernos, 
que se revisen periódicamente en función de los acontecimientos macroeconómicos y financieros. Es decir, que se ponga en marcha un sistema de evaluación de riesgo sistémico, lo que se conoce como la supervisión macroprudencial. Además de esta supervisión macroeconómica, debe establecerse una regulación microeconómica, basada en aspectos como:

1. Límites al crecimiento rápido del crédito y a la concentración de riesgos. Estos últimos existen pero es un aspecto en el que claramente, los bancos centrales podían haber hecho más. El crecimiento del crédito en sus diversas finalidades debe ser un objetivo intermedio de la política monetaria.

2. Limitar el apalancamiento de las instituciones financieras y el establecimiento de estándares de rating por tipos de crédito.

3. El sistema de pago a las agencias de rating debe cambiar siendo los inversores, y no los emisores, quienes lo hagan.

4. Debe prohibirse la toma de posiciones propias (proprietary trading) por parte de las entidades financieras, por el conflicto de intereses que genera respecto de la toma de posiciones en nombre de clientes y por el riesgo que entraña para el capital de la institución.

5. Quizá sea conveniente ajustar los requisitos de reservas al nivel de riesgo incorporado en la estructura de capital del banco.

6. Asimismo, es preciso requerir una mayor difusión de información por parte de hedge funds y fondos de inversión privada. La regla debe ser que los agentes a quienes se ofrezca un instrumento financiero deben estar en condiciones de comprender perfectamente su naturaleza, y el riesgo que asumen.

7. Derivados complejos como los credit default swaps deberían ser negociados en mercados organizados con cámaras de compensación y plenamente respaldados por una garantía colateral.

8. Deben eliminarse los incentivos a la compra de viviendas, que se han mantenido por demasiado tiempo por parte de distintos gobiernos. Un subsidio fuerte a la compra de viviendas, que implica una transferencia de renta entre contribuyentes.

Todo esto son elementos para proteger al pequeño inversor, pero también son instrumentos de política macroeconómica. Dos elementos regulatorios merecen consideración aparte. Por un lado, el tratamiento de las compensaciones salariales (bonus) a los altos ejecutivos de entidades financieras, algunas de las cuales han estado en el centro de las irregulares prácticas causantes de la crisis, por lo que han creado una justificada alarma social. Por otro, el diseño que queremos para el sector financiero futuro; en particular, si es posible y conveniente mantener una grado de heterogeneidad regulatoria compatible con un grado suficiente de competencia y con la estabilidad financiera.

Limitar el nivel y estructura de la compensación a los altos ejecutivos puede dar pie a todo tipo de escapatorias legales, a la vez que puede distorsionar el mercado. 
Por otro lado, cualquier intervención de este tipo debería llevarse al nivel de quienes llevan a cabo la negociación de activos, pues son quienes asumen posiciones de riesgo, por lo que deben tener los incentivos adecuados y mecanismos de supervisión de riesgos adecuados. Las verdaderamente ingentes cifras de compensación salarial a empleados no corresponden solo a altos ejecutivos. Pero es imposible que el gobierno regule la remuneración de todos los empleados de una institución financiera. Es cierto que en algunos casos son alarmantes, pero es preferible potenciar que los accionistas dispongan de información detallada al respecto y que puedan presentar sus reclamaciones en los foros apropiados (Krugman, 2009).

Son razones que hacen pensar que no procede tratar de regular el nivel salarial de ningún colectivo profesional, lo cual no es incompatible con una elevación drástica de los tipos impositivos para rentas muy altas.

En todo caso, la teoría económica nos enseña que unos incentivos adecuados requieren que la compensación salarial a) sea simétrica, es decir, dependiente de los resultados tanto cuando son buenos como cuando son malos, b) esté basada en los resultados derivados para la empresa a largo plazo de la actuación profesional. Para ello, las compensaciones anuales deberían mantenerse en un fondo que va acumulando resultados, y del que solo pueden extraerse con una fuerte penalización si el trabajador abandona la empresa. O puede pagarse la compensación en acciones de la empresa que no pueden venderse hasta transcurrido un tiempo suficiente para aflorar los resultados de la gestión del trabajador. Y, en todo caso, sería conveniente extremar la transparencia en la evaluación económica de todas las formas de remuneración de los ejecutivos.

Uno de los grandes interrogantes que surgen al plantear reformas regulatorias es el tratamiento que se debe dar a las instituciones que no son bancos comerciales, como algunos hedge funds o la banca de inversión antes de la crisis, pero que ofrecen servicios comparables a los de la banca comercial tradicional, como la emisión de cheques. El mundo de la intermediación financiera es extremadamente complejo en la actualidad, y la banca tradicional llevaba a cabo solo un porcentaje del mismo antes de la crisis. Si se somete a todas estas instituciones al mismo régimen regulatorio que la banca comercial, puede representar el fin de las mismas, como es el caso de la banca de inversión. Por otra parte, si se permite una brecha regulatoria entre distintos tipos de instituciones se distorsiona la competencia entre ellas. Precisamente la enorme heterogeneidad de la industria financiera magnifica la dificultad de diseñar y poner en práctica un sistema regulatorio universal. Podríamos pensar en volver a la tradicional banca comercial financiada mediante depósitos y emisora de préstamos comerciales a corto plazo. Pero este tipo de banca no es viable si se permite otro tipo de intermediario financiero con productos sustitutivos. ¿Debemos entonces de obligar a los hedge funds, money market funds y bancos no comerciales a cumplir requisitos de reservas? Este es un problema de difícil solución, que requerirá un análisis muy cuidadoso en los próximos años. 


\subsection{Marco teórico del bail-out vs. bail-in}

Uno de los elementos fundamentales de una reestructuración o resolución bancaria es la determinación de quién asume las pérdidas y, en caso de que el banco vaya a continuar su actividad, quién aporta los fondos para la recapitalización. Hay tres posibilidades básicas:

- Los propios accionistas y acreedores del banco (bail-in).

- El presupuesto público (rescate público o bail-out).

- Fondos financiados con aportaciones del sector bancario (Fondo de Garantía de Depósitos -FGD- o Fondo de Resolución).

En el caso de crisis en empresas del sector real de la economía, cuando no logran refinanciar su deuda y se ven abocadas al concurso de acreedores, son los accionistas y acreedores los que sufren las pérdidas (lo que para el sector bancario hemos llamado bail-in). Sin embargo, en muchas crisis financieras, y en particular en la actual, esta opción ha sido residual.

A nivel internacional, el modelo generalizado en la actual crisis ha sido, mayoritariamente, el del rescate público, protegiendo a los acreedores de los bancos, y no solo para grandes bancos sino también para bancos pequeños.

La razón esencial que ha justificado este trato diferencial del sector financiero a través de un recurso masivo a fondos públicos ha sido la naturaleza especial de los pasivos bancarios. La aplicación de quitas a los instrumentos de financiación de los bancos puede ser desestabilizadora, especialmente si se aplica a algunos pasivos sensibles como los depósitos, la deuda senior o los pasivos por derivados. Comenzando por los depósitos, estos pueden ser de varios tipos. Los más comunes recogen ahorros minoristas que están cubiertos por el FGD (hasta 100.000 euros en la UE), pero incluso con la protección de dicho Fondo, la parte de los depósitos por encima de los 100.000 euros teóricamente sí podría sufrir pérdidas, por lo que en momentos de crisis, si se percibe ese riesgo, puede haber fuertes salidas de depósitos en el conjunto del sistema. Los depósitos pueden ser también de pymes o empresas del sector real, con importes muy superiores a los 100.000 euros y que se utilizan para la gestión de las operaciones corrientes y pago de sueldos. La quita en esos depósitos puede arrastrar al sector real. Por otro lado, otro pasivo sensible desde el punto de vista de estabilidad financiera es la financiación mayorista senior (bonos no garantizados). En caso de que los bonos senior sufran pérdidas, la financiación mayorista puede cerrarse inmediatamente no solo para el banco afectado sino para el conjunto del sector, provocando un efecto contagio. Igualmente, el bail-in a los pasivos por derivados puede generar un efecto arrastre al conjunto del sector.

Por ello, en general, la asignación de pérdidas al sector privado ha sido nula o limitada a los accionistas o, como mucho (caso español), también a los tenedores de deuda subordinada. En definitiva, ante una crisis sistémica la mayoría de los países han preferido optar por salvaguardar la estabilidad financiera aun a costa de recurrir 
al dinero público para socializar pérdidas de una empresa privada. Hay que recordar que, en esos casos, los beneficiarios del bail-out son los financiadores del banco que, en caso de que se hubiera recurrido a la liquidación ordinaria, hubieran sufrido pérdidas en sus títulos (es decir, los depositantes o los acreedores senior). En todo caso, el rescate debería ir siempre acompañado de una evaluación de la responsabilidad de los gestores involucrados en la crisis del banco rescatado.

Aunque en esta crisis haya prevalecido la recapitalización pública, eso no quiere decir que en el futuro ese deba ser el modelo óptimo. De hecho, el FSB (brazo financiero del G20) y el Fondo Monetario Internacional (FMI) defienden que las futuras resoluciones deben basarse fundamentalmente en el bail-in y ello por varias razones:

Los problemas de una empresa privada no deben ser cargados al contribuyente. Si la empresa en cuestión (una entidad financiera en este caso) es tan grande o sistémica que su quiebra pudiera ser más grave para el presupuesto que su rescate (too big to fail), entonces se debería garantizar ex ante que no haya empresas de ese perfil o que el sector público cuente con instrumentos para gestionar sus crisis sin necesidad de nacionalizarlas.

Ningún sector económico (por relevante que sea) debería contar con una garantía implícita del sector público que les asegure que nunca puedan quebrar. Esa garantía implícita sí se ha percibido para el sector financiero y provoca serias distorsiones: por un lado, abarata la financiación al sector bancario no por su calidad relativa, sino en virtud de esa garantía; por otro lado, incentiva comportamientos de riesgo, ya que son una «apuesta de una sola dirección» (en caso de beneficio, es privado; en caso de pérdida, se socializa).

Por estas razones, el FSB, en sus Key Attributes of Effective Resolution Regimes, considera el bail-in la pieza fundamental. No obstante, no todos los analistas comparten la conveniencia del bail-in como modelo general de resolución y existe una corriente que considera que, especialmente para crisis sistémicas, un modelo mixto con bail-in de accionistas y acreedores junior (pero sin llegar a deuda senior ni a depósitos) combinado con bail-out es la mejor opción. En general, esta corriente valora el atractivo teórico del bail-in (disciplina de mercado, protección del contribuyente, etcétera) pero lo considera inviable por su impacto desestabilizador financiero y real que, a la postre, podría ser más gravoso en términos reales y presupuestarios. Es decir, quizá el enfoque bail-in puede funcionar para una entidad pequeña en una crisis idiosincrática y aislada, pero para los demás casos, el óptimo social es el bail-out (o como mucho bail-in de accionistas y acreedores junior). El argumento es que, una vez que se produce una crisis, o el sector público estabiliza la situación o los mercados financieros se paralizarán y cortarán la financiación, retroalimentando la crisis, propagándola y agravándola. Por tanto, concluyen, el argumento del ahorro para el contribuyente es ficticio porque la realidad será mucho más gravosa (FMI, 2008).

El debate se complica porque la alternativa del bail-out, aparte de las desventajas teóricas ya señaladas, también tiene serias dificultades prácticas. El uso de dinero público para el rescate bancario produce gran controversia en la sociedad, más aún en un contexto de crisis económica, por lo que el recurso al rescate tiene 
también condicionantes políticos que pueden llevar a intentar minimizar el importe del apoyo, de modo que no se solucione completamente el problema Valle, 2008). Asimismo, en una crisis sistémica, el dinero público necesario para el rescate puede no ser financiable en el mercado, transformando la crisis bancaria en una crisis de deuda soberana. De hecho, esta es la causa del círculo vicioso en la financiación soberana-bancaria que ha llevado a países europeos a necesitar financiación de la UE para recapitalizar sus sectores financieros o para poder financiar a su sector público.

\section{Medidas propuestas por el Consejo de Estabilidad Europea}

Dentro de las medidas propuestas por el Consejo de Estabilidad Europea establece como objetivos:

- Asignar pérdidas a accionistas y acreedores sin garantías adicionales, respetando el orden de prelación de pasivos.

- Proteger, en todo caso, los depósitos cubiertos (hasta 100.000 euros en Europa), pues los fondos de protección de depósitos los garantizan.

- No depender de apoyo público. Del modelo del FSB se desprende que el bailout, de producirse, debería ser solo en última instancia. El principio de minimización del coste para el contribuyente tiene dos dimensiones: que se utilice el menor dinero público posible y que, una vez que haya sido inevitable utilizar dinero público, se intente maximizar la recuperación.

- Extender el bail-in a los acreedores sénior.

- Crear en cada país un fondo de resolución financiado con contribuciones del propio sector y al que se pueda recurrir para aportar liquidez y financiación (Valle, 2008). Este fondo, en lo que afecta al objeto de este artículo, se utilizaría para absorber pérdidas en lugar de algún pasivo al que se considera conveniente proteger del bail-in. Es decir, el fondo de resolución cumple un doble papel: en primer lugar, evita los efectos perniciosos del bail-in, al proteger pasivos especialmente sensibles (por ejemplo, permite proteger los depósitos de personas naturales o de pymes más allá de lo cubierto por el FGD); y, en segundo lugar, evita tener que recurrir al bail-out al ser el conjunto del sector financiero el que asume esos costes. En definitiva, el Fondo de Resolución es un instrumento muy útil para «cuadrar el círculo» pues puede mitigar tanto el bail-in como el bail-out. No obstante, el recurso a ese Fondo tampoco puede ser ilimitado, ya que su dotación depende de las aportaciones de los bancos y, por tanto, también afecta a sus cuentas. Asimismo, también relaja la disciplina bancaria si su uso no se limita estrictamente a financiar la exclusión de los pasivos más sensibles. 


\section{El semestre europeo y su relación con las políticas económicas y de empleo}

La reciente crisis y la puesta en práctica de excepcionales medidas de salvamento han aflorado múltiples cuestiones en las que el mundo académico debe hacer claros progresos. Comenté antes acerca de la excesiva simplicidad que aún presentan los modelos monetarios que actualmente sirven de referencia en los principales bancos centrales, especialmente por la gran complejidad que ha adquirido muy rápidamente el sector financiero, y por su definitivo entronque con la economía real, aspectos estos apenas incorporados en los modelos mencionados. También es importante que avancemos en entender la relevancia que para la política fiscal tiene la composición del gasto, las distorsiones creadas por distintos impuestos, y cuál es la eficacia relativa de las políticas de gasto respecto de las políticas impositivas o de transferencias. En el diseño de políticas fiscales de expansivas, es importante que entendamos los condicionantes que determinadas medidas a corto plazo tienen sobre el crecimiento a largo plazo (FMI, 2008).

Una caída transitoria del producto, incluso si es cuantiosa, tiene menor coste social que una medida que limite en una pequeña cuantía el crecimiento durante un número de períodos. Este es uno de los principales argumentos por los que las políticas de gasto deben diseñarse cuidadosamente en función de su efecto sobre el crecimiento. La acumulación de capital productivo, la inversión en nuevas tecnologías, la innovación, la acumulación de capital humano y la educación deben tener carácter prioritario, frente a subvenciones no finalistas (Morris et al., 2006).

Es imprescindible progresar definitivamente en una regulación de los mercados de trabajo que evite sus ineficiencias, especialmente en países como España, y que permita reducir la volatilidad del empleo a lo largo del ciclo económico (Pernias, 2013). En Europa, sería deseable que tal legislación pudiera adoptarse a nivel del área monetaria, lo cual no es sino síntoma de la conveniencia de disponer de un área económica y financiera común en apoyo de una moneda única.

El semestre europeo incluye el control de las políticas económicas y de empleo, los programas de estabilidad, los programas nacionales de reforma y los desequilibrios macroeconómicos, para lo que se introduce la posibilidad de que el Consejo, tras evaluar los programas, dirija a los EEMM «orientaciones obligatorias» de cara a los presupuestos nacionales del ejercicio siguiente, y que la Comisión realice controles reforzados in situ sobre el grado de cumplimiento de los compromisos adquiridos. Ante el incumplimiento de tales recomendaciones, la reacción, como es habitual, se gradúa mediante tres pasos sucesivos: nuevas recomendaciones, advertencia de la Comisión, y aplicación de las medidas del Reglamento 1.176/2011.

La reforma de 2011 incluye también otras determinaciones respecto de los objetivos de déficit y deuda propiamente dichos. En este sentido, se proclama que cada Estado miembro tendrá un objetivo a medio plazo que \{garantizará un rápido avance hacia la sostenibilidad de las finanzas públicas», y se señala como límite del déficit estructural (ajustado al ciclo y tenidas en cuenta las medidas temporales) del 1 por 
100, si bien podrá superarse en caso de acontecimientos fuera del control del Estado o en periodos de crisis económica grave (Pernias, 2013).

Con relación a los programas de estabilidad, las nuevas normas señalan que deberán contener las siguientes menciones:

1. El objetivo presupuestario a medio plazo.

2. La trayectoria de ajuste del déficit y de la deuda.

3. La evolución prevista de los gastos e ingresos.

4. La cuantificación de las medidas discrecionales de ingresos, y de las obligaciones implícitas relacionadas con el envejecimiento de la población y otras cuestiones.

\section{Conclusión}

La política monetaria debe perseguir la estabilidad de precios como elemento necesario, aunque no suficiente, para lograr un crecimiento sostenido y el mantenimiento de un desempleo reducido. La política fiscal debe perseguir el mayor saneamiento posible del sector público, reduciendo la ratio deuda sobre PIB, lo que permitirá mayor margen de maniobra cuando surjan posibles dificultades. La política monetaria debe prestar atención a múltiples indicadores de distintos mercados, como los niveles de apalancamiento o el comportamiento de los precios de determinados activos financieros y reales, abandonando la idea de que la conexión entre mercados permite, mediante arbitraje, la simplificación al máximo del diseño de política monetaria. Tiene a su alcance una multiplicidad de elementos regulatorios que puede utilizar discrecionalmente para evitar la ocurrencia de burbujas en los precios de activos financieros y reales. La regulación financiera debe insertarse como componente indispensable de la política monetaria. En modo alguno podemos volver a caer en

el error de identificar mercados libres con mercados desregulados.

La extrema agresividad de la crisis ha puesto a todos de acuerdo. Posiblemente por primera vez desde la creación de la Unión Europea, no hay apenas desavenencias sobre el diagnóstico de la situación económica, sobre los objetivos a lograr -independientemente del perfil político de los gobiernos- ni sobre la relación medios-fines, con un consenso generalizado alrededor de la preferencia por las políticas de gasto frente a las políticas impositivas.

En la fase alcista del ciclo económico, los progresos hacia la estabilidad fiscal fueron generalizados; pero cuando los principales países de la Unión pasaron por dificultades (Francia y Alemania en 2003) la respuesta consistió en la modificación del Pacto para evitar la imposición de sanciones. Está por ver si en su actual formulación, el PEC será capaz de digerir el notable deterioro que están experimentando las cuentas públicas de prácticamente todos los países de la Unión o, si por el contrario, será necesaria una nueva reformulación del mismo que podría llevar, en el límite, a un cambio estructural en la «gobernanza» de la política fiscal desde la perspecti- 
va de la coordinación en el marco de la UE, con la búsqueda de nuevos instrumentos.

El reparto del impacto sobre el déficit público entre los estabilizadores automáticos y las políticas discrecionales varía entre países. El tamaño del sector público en la economía de cada país así como la propia estructura productiva determinan generalmente el peso anti cíclico de los estabilizadores. A su vez, su importancia acota el margen de maniobra de que disponen los gobiernos para las políticas fiscales discrecionales.

La reestructuración del sector financiero en España ha supuesto un coste importante que ha sido repartido entre el sector público y el sector privado. Dada la regulación sobre resolución actualmente en tramitación a nivel europeo, la tendencia se dirige hacia una involucración creciente del sector privado en las pérdidas de modo que la participación pública, de haberla, sea residual y, en todo caso, de última instancia.

Esto debe proteger al contribuyente y asimismo debería fomentar una mayor disciplina en la gestión bancaria. Sin embargo, obliga también a que el sistema financiero sea capaz de soportar una resolución de este tipo sin sufrir efectos desestabilizadores que acaben resultando más dañinos para la economía en su conjunto que si se hubiera optado por el bail-out. En este sentido, hay varios elementos claves para que el sector financiero esté correctamente preparado para ese modelo:

En primer lugar, que las reglas sean conocidas ex ante. A futuro, todos los agentes deben ser conscientes de que la garantía implícita del sector público a los bancos se ha reducido considerablemente.

Que las entidades financieras tengan en su pasivo suficientes elementos para garantizar una capacidad de absorción de pérdidas suficiente. Es decir, deben contar con capital, deuda subordinada y/o deuda sénior suficiente para que, en caso de pérdidas, estas no alcancen rápidamente a los depósitos.

Que exista un fondo de resolución financiado por el sector que permita que determinados pasivos, por su especial sensibilidad (fundamentalmente los depósitos de pymes y personas físicas -en la parte superior a 100.000 euros-) puedan ser excluidos del bail-in sin que eso inmediatamente obligue a llegar al bail-out. En este sentido, la utilidad de este fondo aumentaría considerablemente si en el contexto de la unión bancaria se creara un fondo único para el conjunto de los países participantes. La propuesta que ha realizado la Comisión así lo recoge pero todavía está negociándose, siendo este uno de los elementos fundamentales.

Que, en los momentos de estabilidad, se realicen previsiones sobre cómo se resolvería cada entidad financiera en caso de entrar en problemas. Es decir que se lleven a cabo análisis sobre la «resolubilidad» de cada institución que permitan detectar ex ante posibles obstáculos a una eventual resolución y que puedan ser abordados antes de que la entidad entre en problemas. 


\section{Referencias bibliográficas}

[1] BCE (2009). «Impacto de las finanzas públicas de la zona del euro del apoyo de los gobiernos al sector financiero». Boletín Mensual, julio, 69-82. Disponible en http://www. bde.es/webbdeles/secciones/informes/Publicaciones_de/Boletin_Mensual_/anoactual/. Recuperado 15-02-16.

[2] CARPIO, M. (2004). «La revisión del Pacto de Estabilidad y Crecimiento». IGAE, 9, 43-45, marzo. Recuperado 08-02-16.

[3] COMISIÓN EUROPEA (2008). «Un Plan Europeo de Recuperación Económica». Comunicación de la Comisión al Consejo Europeo, noviembre, Bruselas. Disponible en http://eurlex.europa.eu/LexUriServ/LexUriServ.do? uri=COM:2008:0800:FIN:ES: $P D F$. Recuperado 08-02-16.

[4] COMISIÓN EUROPEA (2009). Economic Forecast. Primavera. Dirección General de Asuntos Económicos y Financieros. Disponible en http://ec.europa.eu/economy_finance/publications/specpub_list12526.htm. Recuperado 08-02-16.

[5] DEROOSE, S.; LARCH, M. y SCHAECHTER, A. (2008). «Constricted, lame and procyclical? Fiscal policy in the euro area revisited». Economic Papers, 353, Comisión Europea.

[6] FMI (2008). «Fiscal policy as a countercyclical tool». World Economic Outlook, octubre, pp. 159-196.

[7] HÜFNER, M. (2004). «La revisión del Pacto de Estabilidad y Crecimiento». IGAE, 9, 39-42, marzo.

[8] KNAPPE, K. y WEBER, M. (2007). «Fiscal policy in EMU after the reform of the European Stability and Growth Pact». CESifo Forum, invierno. ABI/INFORM Global, 45-51.

[9] KRUGMAN, P. (2009). "Misión no cumplida». El País, 4 de octubre.

[10] MATEOS DE LA NAVA, I. (2009). «El cambio de paradigma de la política fiscal y su reflejo en el Pacto de Estabilidad y Crecimiento». Tesis doctoral presentada en marzo en la Universidad Autónoma de Madrid.

[11] MORRIS, R.; ONGENA, H. y SCHUKNECHT, L. (2006). «The reform and implementation of the Stability and Growth Pact». Occasional Paper, 47, junio. BCE disponible en http://www.ecb.int/pub/scientific/ops/date/html/ops2006.en.html. Recuperado 04-0216.

[12] MUNDELL, R. A. (1960). «The Monetary Dynamics of International Adjustment Under Fixed and Flexible Exchange Rates». Quarterly Journal of Economics, 84 (2), 227 57.

[14] PERNIAS, S. (2013). «La quita y la conversión de los acreedores bancarios». El bail-in, IEB.

[15] VALLE, V. (2008). «¿Es la hora de la política fiscal?». Cuadernos de Información Económica, nov.-dic. Madrid. Fundación de las Cajas de Ahorros. 